\title{
Bone apposition to titanium implants biocoated with recombinant human bone morphogenetic protein 2 (rhBMP-2). A pilot study in dogs
}

\author{
Jürgen Becker • Axel Kirsch • Frank Schwarz • \\ Maria Chatzinikolaidou • Daniel Rothamel • \\ Vojislav Lekovic • Markus Laub • \\ Herbert Peter Jennissen
}

Published online: 27 July 2006

(C) Springer-Verlag 2006

\section{Clin Oral Invest (2006) DOI 10.1007/s00784-006-0049-0}

The name of one of the authors, Markus Laub, was omitted. The complete list is given above.

The third address was incorrect. The correct version is given below.

The online version of the article can be found at http://dx.doi.org/ 10.1007/s00784-006-0049-0.

J. Becker $(\bowtie) \cdot$ F. Schwarz $\cdot$ D. Rothamel

Department of Oral Surgery, Heinrich-Heine-University,

Düsseldorf, Germany

e-mail: jbecker@uni-duesseldorf.de

A. Kirsch

Private Practice,

Filderstadt, Germany

M. Chatzinikolaidou $\cdot$ M. Laub $\cdot$ H. P. Jennissen

Institute for Physiological Chemistry,

University of Duisburg-Essen,

Hufelandstraße 55, 45122 Essen, Germany

\section{Lekovic}

Department of Periodontology, University of Belgrade,

Belgrade, Serbia and Montenegro 